\title{
Zero-dimensional singular continuous spectrum for smooth differential equations on the torus
}

\author{
A. $\mathrm{HOF} \dagger$ and O. KNILL $\ddagger$ \\ Division of Physics, Mathematics and Astronomy, California Institute of Technology \\ 253-37, Pasadena, CA 91125, USA
}

(Received 14 September 1996 and accepted in revised form 24 July 1997)

\begin{abstract}
We study spectral properties of the flow $\dot{x}=1 / F(x, y), \dot{y}=1 / \lambda F(x, y)$ on the 2 -torus. We show that, in general, the speed of approximation in cyclic approximation gives an upper bound on the Hausdorff dimension of the supports of spectral measures. We use this to prove that for generic pairs $(F, \lambda)$ the spectrum of the flow on the torus is singular continuous with all spectral measures supported on sets of zero Hausdorff dimension.
\end{abstract}

1. Introduction

On the torus $\mathbb{T}^{2}:=\mathbb{R}^{2} / \mathbb{Z}^{2}$ consider the flow $T_{t}$ defined by

$$
\frac{d x}{d t}=\frac{1}{F(x, y)}, \quad \frac{d y}{d t}=\frac{1}{\lambda F(x, y)} .
$$

It is known that for 'most' real analytic $F>0$ there exist $\lambda$ such that the flow has purely singular continuous spectrum (see Chapter 16 in [1]; the presentation there is based on results of Kolmogorov [13], Shklover [20] and Katok [9]). It is also known that for Lebesgue almost all $\lambda$ the spectrum is discrete if $F \in \mathcal{C}^{5}$. Thus small perturbations in $\lambda$ can render the spectrum of the flow either continuous or discrete.

This raises two questions. How exceptional is singular continuous spectrum for these flows? What can be said about the Hausdorff dimension of the supports of the spectral measures when the spectrum is singular continuous?

These questions are motivated in part by analogous questions in the theory of Schrödinger operators $[\mathbf{2 1}, \mathbf{2}]$. There exist one-parameter families $H_{\lambda}$ of Schrödinger operators that have purely discrete spectrum for almost all $\lambda$ and purely singular continuous spectrum for generic $\lambda$ (i.e. for $\lambda$ in a dense $G_{\delta}$ ). But this spectral instability is not as dramatic as it first seems, because for all $\lambda$ all spectral measures are supported on sets of Hausdorff dimension zero [2, Theorem 7.4].

$\dagger$ Current address: NCM, PO Box 473, 1000 AL Amsterdam, The Netherlands.

$\ddagger$ Current address: Department of Mathematics, University of Arizona, Tucson, AZ 85721, USA. 
In $\S 2$ we show that singular continuous spectrum is in fact common: it occurs for generic pairs $(F, \lambda)$. The proof is based on Simon's wonderland theorem [21] and a small change to the proof presented in [1]. In $\S 4$ we show that the spectrum is generically zero dimensional. The proof uses a result of Strichartz in Fourier theory [23], new spectral decompositions of self-adjoint operators [16], and elements of the theory of periodic approximations $[\mathbf{1 0}, \mathbf{1}]$. These two results show that the spectrum is generically singular continuous and zero dimensional. We do not know if the spectral measures are supported on sets of Hausdorff dimension zero for all $\lambda$.

As an intermediate result we show in $\S 3$ that, in full generality, the speed of approximation of a flow in a cyclic approximation provides an upperbound on the Hausdorff dimension of the support of spectral measures. The discrete time analogue gives a weak version of a recent result of Iwanik [8].

Dimensional properties of spectral measures of dynamical systems are of interest for a number of reasons. First, it is natural to study them. Next, in particular examples, as in the flows on the torus we study here, the fact that spectral measures are supported on sets of zero Hausdorff dimension sheds new light on the transition from discrete to singular continuous spectrum. Finally, those dimensional properties are related to rates of decay of (averaged) correlation functions [12] and to critical exponents of generalized random walks [7].

Flows defined by differential equations of the form (1) are not as special as they may seem. If $A, B \in \mathcal{C}^{5}\left(\mathbb{T}^{2}\right)$ and the flow defined by

$$
d x / d t=A(x, y), \quad d y / d t=B(x, y)
$$

has no fixed points and has an invariant measure that is absolutely continuous with respect to Lebesgue measure with a density that can be chosen to be $\mathcal{C}^{5}$, then (2) reduces to (1) by a change of coordinates ([22], or $\S 16.1$ in [1]).

If $F$ is not smooth then the flows defined by (1) can be strongly mixing [14]. The flow can also have mixed continuous-discrete spectrum, for continuous $F[\mathbf{1 5}]$ and even for analytic $F[3]$.

\section{Singular continuous spectrum is generic}

There are four main steps in the proof in [1] that there exist real analytic functions $F$ for which there are $\lambda$ such that $T_{t}$ has purely singular continuous spectrum. First, the flow $T_{t}$ can be represented as a flow $S_{t}^{f, \lambda}$ under a function $f>0$ over the rotation $R_{\lambda} u=u+\lambda$ on $\mathbb{T}$, where

$$
f(u)=\lambda \int_{0}^{1} F(u+\lambda \xi, \xi) d \xi
$$

[1, p. 435]. Second, if $f$ is $\mathcal{C}^{5}$ (which is the case if $F \in \mathcal{C}^{5}\left(\mathbb{T}^{2}\right)$ ), then $S_{t}^{f, \lambda}$ has purely discrete spectrum for Lebesgue almost all $\lambda[1$, Theorem 16.2.1] and for all $\lambda \in \mathbb{T}$, the spectrum is singular with respect to Lebesgue measure [1, Theorems 16.3.1 and 15.4.3]. Third, for every real analytic $f>0$ which is not a trigonometric polynomial there exist $\beta \in \mathbb{T}$ such that the flow $S_{t}^{f, \beta}$ is weakly mixing $[1, \S 16.4]$. That means that the spectrum of $S_{t}^{f, \beta}$ is continuous; by the second statement it is then purely singular continuous. 
Fourth, if $f(u)=\sum_{k \in \mathbb{Z}} f_{k} e^{2 \pi i k u}$ is the Fourier series of the real analytic function $f$ on $\mathbb{T}$ then for all $\lambda \in \mathbb{R}(3)$ has a real analytic solution $F>0$ if

$$
f_{0}>\frac{\pi}{2} \sum_{k \neq 0}\left|f_{k}\right|
$$

[1, Lemma 16.4.1]. Thus there exist real analytic $F$ for which the flow $T_{t}$ has purely singular continuous spectrum for some $\lambda$.

It is also clear now that for 'most' real analytic $F>0$, there are $\lambda$ for which $T_{t}$ has purely singular continuous spectrum. For if $F>0$ is real analytic then so is $f>0$; 'most' real analytic $F$ have infinitely many non-zero Fourier coefficients and 'typically' those will not be such that $f$ is a polynomial. In any reasonable topology 'most' will certainly encompass a dense set.

The small change to a proof in [1] is in the third step.

LEMMA 2.1. If a real analytic $f>0$ is not a trigonometric polynomial then there is a dense set $B \subset \mathbb{T}$ such that for $\beta \in B$ the flow $S_{t}^{f, \beta}$ is weakly mixing.

Proof. It is easy to see that in Lemma 16.4.3 of [1] one can impose the condition that the rational numbers $\beta_{n} \rightarrow \beta$ are all contained in any given interval. This gives that $T_{t}^{f, \beta}$ is weakly mixing for a dense set of $\beta$.

The wonderland theorem [21] can be formulated as follows. Let $\mathcal{X}$ be a complete metric space of self-adjoint operators on a separable Hilbert space for which convergence in the metric implies strong resolvent convergence. Suppose the sets of operators in $\mathcal{X}$ that have purely continuous spectrum and purely discrete spectrum are dense in $\mathcal{X}$. Then there is a generic set in $\mathcal{X}$ (i.e. a dense $G_{\delta}$ ) of operators that have purely singular continuous spectrum. It is straightforward to apply the wonderland theorem to a flow under a function over a rotation of the circle [6].

PROPOSITION 2.2. If a real analytic $f>0$ is not a trigonometric polynomial then the flow $S_{t}^{f, \alpha}$ has purely singular continuous spectrum for generic $\alpha$.

Proof. Let $M_{f}:=\{(x, y) \mid x \in \mathbb{T}, 0 \leq y<f(x)\}$. Denote the normalized Lebesgue measure on $M_{f}$ by $\mu$; it is invariant under $S_{t}^{f, \alpha}$ for every $\alpha$. Let $\mathcal{H}^{\perp}:=\left\{\phi \in \mathrm{L}^{2}\left(M_{f}, \mu\right) \mid\right.$ $\left.\int \phi d \mu=0\right\}$; this is a separable Hilbert space with inner product $(\phi, \psi)=\int \phi \bar{\psi} d \mu$.

Let $U_{t}^{\alpha}$ be the group of unitary operators on $\mathcal{H}^{\perp}$ defined by $U_{t}^{\alpha} \phi:=\phi \circ S_{t}^{f, \alpha}$. By Stone's theorem there exists for each $\alpha$ a self-adjoint operator $L_{\alpha}$ on $\mathcal{H}^{\perp}$ such that $U_{t}^{\alpha}=\exp \left(2 \pi i t L_{\alpha}\right)$. The spectral measures $v_{\phi}^{\alpha}$ of $L_{\alpha}$, defined by $\int e^{2 \pi i \lambda t} d v_{\phi}^{\alpha}(\lambda)=$ $\left(e^{2 \pi i t L_{\alpha}} \phi, \phi\right)$ (see, for example, [18, p. 263]) coincide with the spectral measures of $U_{t}^{\alpha}$. Let $\mathcal{X}:=\left\{L_{\alpha} \mid \alpha \in \mathbb{T}\right\}$ with the metric of $\mathbb{T}$. If $\alpha_{n} \rightarrow \alpha$ in $\mathbb{T}$, then $U_{t}^{\alpha_{n}} \rightarrow U_{t}^{\alpha}$ strongly in $\mathcal{H}^{\perp}$ for each $t$ (as can be seen by looking at characteristic functions of rectangles). This implies that $L_{\alpha_{n}} \rightarrow L_{\alpha}$ in the strong resolvent sense (see, for example, [18, Theorem VII.21]).

By Lemma 2.1, the spectrum of $C^{\alpha}$ is continuous for $\alpha$ in a dense set. As mentioned in the second of the four main steps, it is purely discrete for Lebesgue almost every $\alpha$. Every set of full Lebesgue measure is dense in $\mathbb{T}$. Thus the wonderland theorem gives that $L_{\alpha}$ (and hence the flow $S_{t}^{f, \alpha}$ ) has purely singular continuous spectrum for generic $\alpha$. 
It follows that for every real analytic $F$ on $\mathbb{T}^{2}$ for which the function $f$ defined in (3) is not a trigonometric polynomial the flow $T_{t}$ has purely singular continuous spectrum for generic $\lambda$. The set of generic $\lambda$ depends on $F$. This should be contrasted with the fact that for every real analytic $F$ the flow $T_{t}$ has purely discrete spectrum for Lebesgue almost all $\lambda$ in a set that is independent of $F$. (For $F \in \mathcal{C}^{5}$ the flow has purely discrete spectrum for all $\lambda$ such that $|\lambda-p / q|>C / q^{4}$ for some constant $C$ and all relatively prime integers $p, q[1, \S 16.2]$.) It is not a priori obvious that the flow $T_{t}$ has purely singular continuous spectrum for generic pairs $(F, \lambda)$. Such a statement does follow from the wonderland theorem. For $c>0$ let $\|F\|_{c}:=\sum_{n, m \in \mathbb{Z}}\left|F_{n m}\right| e^{c(|n|+|m|)}$, where the $F_{n m}$ are the Fourier coefficients of $F$ and let $\mathcal{A}_{c}$ be the Banach space obtained by completing the trigonometric polynomials in the norm $\|\cdot\|_{c}$. Then $\mathcal{A}_{c}$ is a complete metric space of real analytic functions.

PROPOSITION 2.3. For generic pairs $(F, \lambda)$ in $\mathcal{A}_{c} \times \mathbb{R}$ (with norm $\max \left(\|\cdot\|_{c},|\cdot|\right)$ ), the flow $T_{t}$ has purely singular continuous spectrum.

Proof. Write $T_{t}^{F, \lambda}$ to stress the dependence of the flow on $F$ and $\lambda$. The measure $F(x, y) d x d y$ is invariant under $T_{t}^{F, \lambda}\left[\mathbf{1}\right.$, p. 435]. Let $\mathcal{H}_{F}:=\mathrm{L}^{2}\left(\mathbb{T}^{2}, F d x d y\right)$ and $\mathcal{H}:=\mathrm{L}^{2}\left(\mathbb{T}^{2}, d x d y\right)$. Consider $U_{t}^{F, \lambda}$, acting on $\mathcal{H}_{F}$ by $U_{t}^{F, \lambda} \phi=\phi \circ T_{t}^{F, \lambda}$. Its spectral measures $v_{\phi}^{F, \lambda}$ are purely singular continuous on $\mathbb{R} \backslash\{0\}$ for a dense set of $(F, \lambda)$ (if $\int \phi F d x d y \neq 0$ they have a point mass at 0 ). They are also purely discrete on $\mathbb{R} \backslash\{0\}$ for a dense set of $(F, \lambda)$. The operator $V_{F}: \mathcal{H}_{F} \rightarrow \mathcal{H}$ defined by $V_{F} \phi:=\phi \sqrt{F}$ is unitary. Let $W_{t}^{F, \lambda}:=V_{F} U_{t}^{F, \lambda} V_{F}^{-1}$ act on $\mathcal{H}$, and denote its generator by $L_{F, \lambda}$. Then the spectral measure of $V_{F} \phi$ for $W_{t}^{F, \lambda}$ is also $v_{\phi}^{F, \lambda}$.

Let $\mathcal{X}:=\left\{L_{F, \lambda} \mid F \in \mathcal{A}_{c}, \lambda \in \mathbb{R}\right\}$ with the metric induced by the norm on $\mathcal{A}_{c} \times \mathbb{R}$. This is a complete metric space of self-adjoint operators on the separable Hilbert space $\mathcal{H}$. Convergence in metric implies strong resolvent convergence (by the argument in the proof of Proposition 2.2). There are dense sets in $\mathcal{A}_{c} \times \mathbb{R}$ for which the spectrum is, respectively, purely singular continuous and purely discrete on $\mathbb{R} \backslash\{0\}$. The wonderland theorem (in the form of Theorem 2.1 of [21]) gives that the spectrum of $L_{F, \lambda}$ is purely singular continuous on $\mathbb{R} \backslash\{0\}$ for generic $(F, \lambda)$. For generic pairs $(F, \lambda)$, therefore, the flow $T_{t}^{F, \lambda}$ has purely singular continuous spectrum on the orthogonal complement of the constant functions in $\mathcal{H}_{F}$.

Unfortunately, Proposition 2.3 does not lead to a genericity result of purely singular continuous spectrum for the systems of the form (2). Changing either $A$ or $B$ can result in a system that has no absolutely continuous invariant measure. Thus there seems to be no way in which a set of systems of the form (2) can be made into a complete metric space to which our results extend.

To conclude this section, let us comment on a difference between genericity of purely singular continuous spectrum for flows and for automorphisms (invertible measurepreserving transformations).

Consider the Lebesgue space $([0,1], d x)$ and the set $\mathcal{B}$ of all automorphisms that leave $d x$ invariant, with the weak topology. Halmos [4] has shown that a generic $T \in \mathcal{B}$ is weakly mixing. Katok and Stepin have shown by cyclic approximation that a generic $T \in \mathcal{B}$ has purely singular spectrum (which can have a discrete part) [10, Theorem 1.1 
and Corollary 3.1]. Together, these results give that a generic $T \in \mathcal{B}$ has purely singular continuous spectrum. (Knill $[\mathbf{1 1}, \mathbf{1 2}]$ has recently pointed out that genericity of purely singular continuous spectrum in $\mathcal{B}$ can be obtained from Halmos [4] and the wonderland theorem, without invoking the theory of cyclic approximation.)

To prove the denseness of the $G_{\delta}$ for which the spectrum is singular, Katok and Stepin rely in their Theorem 1.1 on the conjugacy lemma [5, p. 77]. The conjugacy lemma seems to have no analogue for flows. Thus, for flows, the wonderland theorem is the natural tool to prove genericity of purely singular continuous spectrum.

\section{Cyclic approximation and the dimension of spectral measures}

This section shows that the theory of periodic approximation gives upper bounds on the Hausdorff dimension of spectral measures. The emphasis is on flows. Analogous results for automorphisms are stated and briefly discussed at the end of the section. We start with some definitions.

A flow $T_{t}$ on a Lebesgue space $(X, m)$ allows a cyclic approximation with speed $g(u) \rightarrow 0$ if there exists a sequence of partitions $\left\{C_{j}^{(n)}\right\}_{j=0}^{Q_{n-1}}$, times $t_{n} \in \mathbb{R}^{+}$and automorphisms $S_{n}: C_{k}^{(n)} \rightarrow C_{k+1}^{(n)}$ such that $\sum_{j=1}^{Q_{n}} m\left(T_{t_{n}} C_{j}^{(n)} \Delta S_{n} C_{j}^{(n)}\right)<g\left(Q_{n}\right)$ and $Q_{n} t_{n} \rightarrow \infty$. (The partitions also have to become arbitrarily fine as $n \rightarrow \infty$. This will play no explicit role here. For details, see [1].)

Let $0 \leq \beta \leq 1$. A measure on $\mathbb{R}$ (or on $\mathbb{T}$ ) is called uniformly $\beta$-(Hölder) continuous if there exists a constant $C$ such that $\mu(I)<C|I|^{\beta}$ for all intervals $I$ of length $|I|<1$. The decomposition of a measure $\mu=\mu_{\mathrm{ac}}+\mu_{\mathrm{s}}$ into an absolutely continuous part $\mu_{\mathrm{ac}}$ and a singular part $\mu_{\mathrm{s}}$ can be generalized. For every $\beta$ there is a decomposition $\mu=\mu_{\beta \mathrm{c}}+\mu_{\beta \mathrm{s}}$ into a $\beta$-continuous part $\mu_{\beta \mathrm{c}}$ and a $\beta$-singular part $\mu_{\beta \mathrm{s}}$. A measure $\mu$ is $\beta$-continuous if $\mu(S)=0$ for every Borel set of zero $\beta$-Hausdorff measure and $\mu$ is $\beta$-singular if it is supported on a set $S$ of zero $\beta$-Hausdorff measure. For details, see $[\mathbf{1 9}, \S 3.3]$ or [16].

If $\mu$ is $\beta$-singular for all $\beta>0$, then $\mu$ is called zero dimensional. This is equivalent to the statement that $\mu$ is supported on a Borel set $S$ of zero Hausdorff dimension. We say that a flow $T_{t}$ has zero-dimensional spectrum if all its spectral measures are zero dimensional.

LEMMA 3.1. Let $T_{t}$ be a measure-preserving flow on a compact Borel probability space $(\Omega, \mu)$. If $T_{t}$ does not have zero-dimensional spectrum, then there exist a $\phi \in \mathcal{H}=L^{2}(\Omega)$ and $a \beta>0$ such that $t \mapsto \phi \circ T_{t}$ is smooth and such that the spectral measure $\mu_{\phi}$ is uniformly $\beta$-continuous.

Proof. If $T_{t}$ is not zero dimensional, there exists a $\beta>0$ such that $\mathcal{H}_{\beta \mathrm{c}} \neq \emptyset$ in the orthogonal decomposition [16] $\mathcal{H}=\mathcal{H}_{\beta \mathrm{s}} \oplus \mathcal{H}_{\beta c}$ of $\mathcal{H}$ into $\beta$-singular and $\beta$-continuous vectors. Because uniformly $\beta$-continuous vectors are dense in $\mathcal{H}_{\beta c}$ [16, Theorem 5.2], there exist a $\phi \in \mathcal{H}$ and a $\beta>0$ such that $\mu_{\phi}$ is uniformly $\beta$-continuous. Take a smooth mollifier function $\psi>0$ with support in $(-1,1)$. Define $\tilde{\phi} \in \mathcal{H}$ by $\tilde{\phi}(\omega):=\int_{-1}^{1} \psi(s) \phi\left(T_{-s} \omega\right) d s$. Then $t \mapsto \tilde{\phi} \circ T_{t}=\left(\psi *\left(\phi \circ T_{t}\right)\right)(\cdot)$ is smooth. Since $\hat{\mu}_{\tilde{\phi}}(t)=\left(\tilde{\phi}, U_{t} \tilde{\phi}\right)=\left(\breve{\psi} * \bar{\psi} * \hat{\mu}_{\phi}\right)(t)$, where $\check{\psi}(x):=\psi(-x)$, we have $\mu_{\tilde{\phi}}=|\hat{\psi}|^{2} \mu_{\phi}$, so $\mu_{\tilde{\phi}}$ is uniformly $\beta$-continuous. 
The next proposition generalises a result of Katok and Stepin [1, Theorem 15.4.1].

Proposition 3.2. Let $r \geq 1$. Let $T_{t}$ be a flow that allows a cyclic approximation with speed $g(u)=o\left(u^{-r}\right)$ using times $t_{n}$ and partitions $\left\{C_{j}^{(n)}\right\}_{j=0}^{Q_{n}-1}$. Then for every spectral measure $\mu_{\phi}$

$$
\max _{k=1, \ldots,\left[Q_{n}^{(r-1) / 2}\right]}\left|\hat{\mu}_{\phi}\left(k Q_{n} t_{n}\right)-\|\phi\|_{2}^{2}\right| \rightarrow 0 \quad \text { as } n \rightarrow \infty .
$$

Proof. Theorem 15.4.1 in [1] covers the case $r=1$. The proposition is proved by modifying the proof of Theorem 15.4.1 in [1] as follows. (Note that $\hat{\mu}_{\phi}(s)=\left(\phi, U_{s} \phi\right)$ so that $\hat{\mu}_{\phi}(s) \rightarrow\|\phi\|_{2}^{2}$ if $\left\|U_{s} \phi-\phi\right\|_{2} \rightarrow 0$.)

The first displayed equation in the proof of Theorem 15.4.1 becomes

$$
\begin{aligned}
\left\|U_{k Q_{n} t_{n}} \phi-\phi\right\|_{2} \leq & \left\|\phi\left(T_{k Q_{n} t_{n}}\right)-\phi_{n}\left(T_{k Q_{n} t_{n}}\right)\right\|_{2}+\left\|\phi_{n}\left(T_{k Q_{n} t_{n}}\right)-\phi_{n}\right\|_{2} \\
& +\left\|\phi_{n}-\phi\right\|_{2}=: \Sigma_{1}+\Sigma_{2}^{(k)}+\Sigma_{3} .
\end{aligned}
$$

Now

$$
\begin{aligned}
\Sigma_{2}^{(k)} & =\left\|\phi_{n}\left(T_{k Q_{n} t_{n}}\right)-\phi_{n}\right\|_{2} \\
& \leq\left\|\phi_{n}\left(T_{k Q_{n} t_{n}}\right)-\phi_{n}\left(T_{(k-1) Q_{n} t_{n}}\right)\right\|_{2}+\cdots+\left\|\phi_{n}\left(T_{Q_{n} t_{n}}\right)-\phi_{n}\right\|_{2} \\
& =k \cdot\left\|\phi_{n}\left(T_{Q_{n} t_{n}}\right)-\phi_{n}\right\|_{2}=k \cdot \Sigma_{2},
\end{aligned}
$$

where $\Sigma_{2}$ is as in the proof of Theorem 15.4.1. There it is shown that $\Sigma_{2} \leq$ $2 C \sqrt{Q_{n} g\left(Q_{n}\right)}$ (without using any specific form for $g$ ). Therefore $\Sigma_{2}^{(k)} \leq 2 C k \sqrt{Q_{n} g\left(Q_{n}\right)}$ which goes to zero uniformly in $k \leq Q_{n}^{(r-1) / 2}$ because of the assumption on the speed of approximation.

Let $\mu$ be a bounded measure on $\mathbb{R}$. A theorem of Strichartz [23] states that if $\mu$ is uniformly $\beta$-continuous, then there is a constant $C$ such that

$$
L^{-1} \int_{0}^{L}|\hat{\mu}(t)|^{2} d t \leq C L^{-\beta} .
$$

We say that a countable set $A \subset \mathbb{R}^{+}$(or $\subset \mathbb{N}$ ) has positive $\beta$ density if there is a constant $C$ such that $|\{a \in A \mid a<n\}| \geq C n^{\beta}$ for infinitely many $n \rightarrow \infty$.

THEOREM 3.3. Suppose a flow $T_{t}$ admits a cyclic approximation with speed $g(u)=$ $o\left(u^{-r}\right)$, times $t_{n} \leq M$ and partitions of size $Q_{n}$. Then every spectral measure of the flow is supported on a set of Hausdorff dimension $\leq 2 /(r+1)$.

Proof. (i) Assume there exists a spectral measure supported on a set of Hausdorff dimension $(1-\beta+\epsilon)>(1-\beta)=2 /(r+1)$ and not on any set of smaller Hausdorff dimension. Then that spectral measure has non-zero $(1-\beta+\epsilon)$-continuous part. Hence $\mathcal{H}_{(1-\beta+\epsilon) \mathrm{c}} \neq \emptyset$ in the decomposition $\mathcal{H}=\mathcal{H}_{(1-\beta+\epsilon) \mathrm{c}} \oplus \mathcal{H}_{(1-\beta+\epsilon) \mathrm{s}}$. By the argument in the proof of Lemma 3.1 there exists a $\phi \in \mathcal{H}$ with a spectral measure $\mu_{\phi}$ that is uniformly $(1-\beta+\epsilon)$-continuous. By Strichartz' theorem [23]

$$
L^{-1} \int_{0}^{L}\left|\left(\hat{\mu}_{\phi}\right)(t)\right|^{2} d t \leq C L^{-(1-\beta+\epsilon)}
$$


for some constant $C$.

(ii) The union (over $n$ ) of the sets $\left\{k Q_{n} t_{n} \mid k=1, \ldots,\left[Q_{n}^{(r-1) / 2}\right]\right\}$ has positive $\beta$-density if

$$
\frac{Q_{n}^{(r-1) / 2}}{\left(Q_{n} t_{n} Q_{n}^{(r-1) / 2}\right)^{\beta}} \geq M^{-\beta} Q_{n}^{(r-1) / 2-\beta(r+1) / 2}
$$

does not go to zero as $n \rightarrow \infty$. This is the case if $(r-1) / 2 \geq \beta(r+1) / 2$ and in particular if $(1-\beta)=2 /(r+1)$.

(iii) By Proposition 3.2, $\hat{\mu}_{\phi}(t)$ is close to $\|\phi\|_{2}^{2}$ at the points $k Q_{n} t_{n}$ ( $k=$ $\left.1, \ldots,\left[Q_{n}^{(r-1) / 2}\right]\right)$ if $n$ is large. Let $U_{t}=e^{i A t}$, where $A$ is the possibly unbounded self-adjoint generator of $U_{t}$. By the definition of $\phi$ in the proof of Lemma 3.1, $\phi$ is in the domain of $A$ (as can, for example, be seen from the proof of Stone's theorem in [18, p. 266]). Therefore the derivative of $\hat{\mu}_{\phi}(t)$ is bounded on $\mathbb{R}$ :

$$
\left|\frac{d}{d t}\left(U_{t} \phi, \phi\right)\right|=|(L \phi(t), \phi)|=|(\phi(t), L \phi)| \leq M^{\prime} .
$$

It follows that there is a constant $C^{\prime}$ such that

$$
\int_{0}^{L}|\hat{\mu}(t)|^{2} d t>C^{\prime} L^{\beta}
$$

for infinitely many $L \rightarrow \infty$. This contradicts (6).

Note in step (ii) that information about the speed with which $t_{n} \rightarrow 0$ as a function of $Q_{n}$ would allow a sharper bound on the Hausdorff dimension.

The discrete time analogues of Proposition 3.2 and Theorem 3.3 can be formulated as follows.

PROPOSITION 3.4. If an automorphism $T$ admits a cyclic approximation with speed $g(u)=$ $o\left(u^{-r}\right)$, using partitions of size $Q_{n}$, then every spectral measure $\mu_{\phi}$ satisfies

$$
\max _{k=1, \ldots,\left[Q_{n}^{(r-1) / 2}\right]}\left|\hat{\mu}_{\phi}\left(k Q_{n}\right)-\|\phi\|_{2}^{2}\right| \rightarrow 0 \quad \text { as } n \rightarrow \infty .
$$

THEOREM 3.5. If an automorphism $T$ admits a cyclic approximation with speed $g(u)=$ $o\left(u^{-r}\right)$ then every spectral measure of $T$ is supported on a set of Hausdorff dimension $\leq 2 /(r+1)$.

The proofs of Proposition 3.4 and Theorem 3.5 are analogous to those of Proposition 3.2 and Theorem 3.3. One has to set $t_{n}=1$. A proof of the Fourier series version of Stricharz' theorem is given in [12]. The discrete time version of Theorem 15.4.1 in [1] can be found as Theorem 3.3 in [10].

Iwanik [8] has a result that is stronger than Theorem 3.5. Under a hypothesis that is implied by the hypothesis of Theorem 3.5 he obtains the sharper bound $\leq 1 / r$. His proof is completely different from ours and uses simultaneous Diophantine approximations. He does not discuss flows. Note that Iwanik gives an example of an automorphism with zero-dimensional continuous spectrum to show that his bound $1 / r$ is not optimal. 
4. Zero-dimensional spectrum is generic

We return now to the flows $S_{t}^{f, \alpha}$ and $T_{t}^{F, \lambda}$. The following theorem generalises a result of Katok [9] (see [1, Theorem 16.3.1, p. 426]).

THEOREM 4.1. Let $\tau \geq 0$. Suppose that $f \in \mathcal{C}^{5+\tau}(\mathbb{T})$ and that there exists a sequence $p_{n} / q_{n}$ of irreducible fractions such that

$$
r_{n}:=q_{n}^{4}\left|\alpha-p_{n} / q_{n}\right|=o\left(q_{n}^{-\tau}\right) \quad \text { as } n \rightarrow \infty .
$$

Then the flow $S_{t}^{f, \alpha}$ admits a cyclic approximation with speed $g(u)=o\left(u^{-(2+\tau)}\right)$, partitions of length $Q_{n}=q_{n} m_{n}$ and times $t_{n}=\gamma / m_{n}$, where $m_{n} \rightarrow \infty$ are integers and $\gamma$ is a constant depending only on $f$.

Proof. Theorem 16.3.1 in [1] covers the case $\tau=0$ (note that there $F$ is a function on $\mathbb{T}$, not on $\mathbb{T}^{2}$, as in this paper). There are two things one has to change in the proof of Theorem 16.3.1 in [1]: the choice of the $m_{n} \in \mathbb{N}$, and the statement of Lemma 1 (p. 427). (Our $\gamma$ is the $\beta$ in [1].)

The modified Lemma 1 reads: suppose $f \in \mathcal{C}^{5+\tau}(\mathbb{T})$ and $|\lambda-p / q| \leq 1 / 10 q^{2}$. Then

$$
\left|\sum_{k=0}^{q-1} f(x+k \alpha)-q \gamma\right|<C_{1} q^{2}|\alpha-p / q|+C_{2} q^{-(3+\tau)}=: \Sigma(p, q)
$$

for certain constants $C_{1}, C_{2}$. The proof is a straightforward generalization of the proof of Lemma 1 on pp. 431-432 of [1]. Note that $\Sigma_{n}:=\Sigma_{n}\left(p_{n}, q_{n}\right)=o\left(q_{n}^{-(2+\tau)}\right)$. The right-hand side of the first displayed equation on p. 430 (which is the expression for $g$ ) now becomes $\left(c / \gamma q_{n}^{2}\right)\left(r_{n}+q_{n}^{-(1+\tau)}\right)$.

In the proof in [1] the sequence of reciprocal times $m_{n} \rightarrow \infty$ has to satisfy three conditions, specified on p. 427 and p. 430.

Condition (i) (p. 427) is $m_{n}<q_{n}^{2} \gamma$ (the ' $/$ ' is a typo). This now becomes $m_{n}<q_{n}^{2+\tau} \gamma$.

Condition (ii) (p. 430) is $r_{n} / q_{n}^{2}=o\left(Q_{n}^{-2}\right)$. This becomes $r_{n} / q_{n}^{2}=o\left(Q_{n}^{-(2+\tau)}\right)$, or $r_{n} q_{n}^{\tau} m_{n}^{2+\tau} \rightarrow 0$.

Condition (iii) (p. 430) is $1 / q_{n}^{3}=o\left(Q_{n}^{-2}\right)$. This becomes $1 / q_{n}^{3+\tau}=o\left(Q_{n}^{-(2+\tau)}\right)$, or $q_{n}^{-1} m_{n}^{2+\tau} \rightarrow 0$.

Because of (8) these three conditions can be satisfied by an integer sequence $m_{n} \rightarrow \infty$.

COROLLARY 4.2. Under the hypotheses of Theorem 4.1 all spectral measures of the flow $S_{t}^{f, \alpha}$ are supported on sets of Hausdorff measure $\leq 2 /(\tau+1)$.

Proof. By Theorem 3.3.

COROLLARY 4.3. If $f$ is real analytic and $\alpha$ is a Liouville number then the flow $S_{t}^{f, \alpha}$ has zero-dimensional spectrum.

Proof. Recall that $\lambda$ is Liouville if there exists for every $l$ a sequence $p_{n} / q_{n}$ of irreducible fractions such that $q_{n}^{l} \cdot\left|\lambda-p_{n} / q_{n}\right| \rightarrow 0$. 
We have not shown that the spectrum of $S_{t}^{f, \alpha}$ is continuous if $\lambda$ is Liouville. Recall that the set of Liouville numbers is a dense $G_{\delta}$ (e.g. [17, §2]). Because the intersection of two generic sets is generic, we have the following.

COROLLARY 4.4. If $f$ is real analytic, but not a trigonometric polynomial, then the flow $S_{t}^{f, \alpha}$ has zero-dimensional continuous spectrum for generic $\lambda$.

The corresponding result for the differential equations (1) justifies the title of our paper.

COROLlaRY 4.5. For generic pairs $(F, \lambda)$ in $\mathcal{A}_{\mathrm{c}} \times \mathbb{R}$ the flow $T_{t}^{F, \lambda}$ has purely singular continuous and zero-dimensional spectrum.

As far as we know this provides the first examples of smooth flows with zerodimensional continuous spectrum.

It would be interesting to know whether the spectrum of $T_{t}^{F, \lambda}$ can fail to be zero dimensional.

\section{REFERENCES}

[1] I. P. Cornfeld, S. V. Fomin and Ya. G. Sinai. Ergodic Theory (Grundlehren der mathematischen Wissenschaften in Einzeldarstellungen, 115). Springer, 1982.

[2] R. del Rio, S. Jitomirskaya, Y. Last and B. Simon. Operators with singular continuous spectrum, IV. Hausdorff dimensions, rank one perturbations, and localization. J. Analyse Math. 69 (1996), 153-200.

[3] A. Ya. Gordon. An example of an analytic flow on a torus with mixed spectrum. Teor. Funktsi $i$ Functsional. Anal. i Prilozhen. 33 (1980), 40-45. Engl. transl. Selecta Math. Soviet. 8(4) (1989), 291298.

[4] P. R. Halmos. In general a measure preserving transformation is mixing. Ann. Math. 45 (1944), 786.

[5] P. R. Halmos. Lectures on Ergodic Theory. The Mathematical Society of Japan, 1956.

[6] A. Hof. On a structure intermediate between quasiperiodic and random. J. Stat. Phys. 84 (1996), 309320.

[7] A. Hof. On scaling in relation to singular spectra. Commun. Math. Phys. 184 (1997), 567-577.

[8] A. Iwanik. Approximation by periodic transformations and Diophantine approximation of the spectrum. Ergodic Theory of $\mathbb{Z}^{d}$-Actions (Proceedings of the Warwick Symposium 1993-4). Eds M. Pollicott and K. Schmidt. Cambridge University Press, 1996, pp. 387-401.

[9] A. B. Katok. Spectral properties of dynamical systems with an integral invariant on the torus. Functional Anal. Appl. 1 (1967), 296-305.

[10] A. B. Katok and A. M. Stepin. Approximations in ergodic theory. Russ. Math. Surv. 22 (1968), 77-102.

[11] O. Knill. Unpublished, 1995.

[12] O. Knill. Singular continuous spectrum and quantitive rates of weakly mixing. Discrete and Continuous Dynamical Systems 4 (1998), 33-42.

[13] A. N. Kolmogorov. On dynamical systems with an integral invariant on the torus. Dokl. Akad. Nauk. SSSR, Ser. Mat. 93 (1953), 763-766. Engl. transl. Selected Works of A. N. Kolmogorov, Volume 1: Mathematics and Mechanics. Ed V. M. Tikhomirov. Kluwer Academic Publishers, 1991, pp. 344-348.

[14] A. V. Kočrgin. On the absence of mixing in special flows over the rotation of a circle and in flows on a two-dimensional torus. Sov. Math. Dokl. 13 (1972), 949-952.

[15] A. B. Krygin. An example of a continuous flow on the torus with mixed spectrum. Math. Notes. 15 (1974), 133-136.

[16] Y. Last. Quantum dynamics and decompositions of singular continuous spectra. J. Funct. Anal. 142 (1996), 406-445.

[17] J. C. Oxtoby. Measure and Category, 2nd edn. Springer, 1980. 
[18] M. Reed and B. Simon. Methods of Modern Mathematical Physics I. (Functional Analysis, 141), revised and enlarged edition. Academic Press, 1980.

[19] C. A. Rogers. Hausdorff Measures. Cambridge University Press, 1970.

[20] M. D. Shklover. On classical dynamical systems on the torus with continuous spectrum. Izv. Vyssh. Uchebn. Zaved. Mat. 10 (1967), 113-124. In Russian.

[21] B. Simon. Operators with singular continuous spectrum: I. General operators. Ann. Math. (1995), 131-145.

[22] S. Sternberg. On differential equations on the torus. Amer. J. Math. 79 (1957), 397-402.

[23] R. S. Strichartz. Fourier asymptotics of fractal measures. J. Func. Anal. 89 (1990), 154-187. 